\title{
Production of composite membrane from waste Kappa-Carrageenan and Tilapia (Oreochromis niloticus) fishbone
}

\author{
Jerry Olay ${ }^{1, *}$, Kristine Ambas ${ }^{1}$, Novelyn Glino $^{1}$, and Vicente Rugi Rubi ${ }^{1}$ \\ ${ }^{1}$ Chemical Engineering Department, College of Engineering, Adamson University, 900 San Marcelino Street, Ermita, \\ Manila, 1000, Philippines
}

\begin{abstract}
Kappa-Carrageenan has been widely used as a gelling agent and stabilizer in the food industry. This research is an application of Kappa Carrageenan as polymer membrane with the combination of Hydroxyl Apatite obtained from the fishbone. Different weight percentages of K-Carrageenan and Fishbone were mixed to make a filtering media with the use of Glutaraldehyde as a crosslinking agent. Before the crosslinking, K carrageenan were pretreated with monochloroacetic acid, ethanol and sodium hydroxide to become an alkali-based solution that will be readily mixed with the hydroxyl apatite. Fishbone were converted to hydroxyl apatite through subjection in a high temperature dryer. Polymer membrane were characterized by evaluating its mechanical and physical properties such as elastic resistance, water permeability and mean pore size diameter with the use of force gauge, filtration due to gravity and scanned electron microscopy respectively. Based from the evaluation, composite with $60 \%$ KCarrageenan- $40 \%$ Fishbone displayed the most feasible characteristic of a filtering media such as its mechanical strength and water permeability. Composite with $80 \%$ KCarrageenan-20\% Fishbone has the smallest pore size in which a good characteristic also of a filtering media.
\end{abstract}

\section{Introduction}

Membrane technology is one of the methods used for separation processes. The principle of this process is that the membrane acts as a very specific filter that let water flow through while it catches suspended solids and other substances. [1]. Membranes are classified whether the thin perm selective layer is porous or dense, and by the type of material (organic, polymer, inorganic, metal, etc.) this membrane film is made from [2].

Porous membranes are made of polymers which includes polysulphones, polyacrylonitrile, polypropylene; ceramics which includes alumina, silica, titania, zirconia, zeolites; and micro porous carbons [2].

Recent studies regarding membrane technology is the hybrid of polymer and ceramic membranes. Development of Thin Film Composite (TFC) membrane which focuses in the incorporation of nanoparticles and nanofibers in the substrate with application in desalination is one example of combining two types of porous membrane. Modification of the monomer thin film has impact on the antifouling and biofouling properties, chemical resistance, mechanical properties and thermal stability [3].

In this study, a composite membrane was be produced from the use of kappa-carrageenan and tilapia (oreochromis niloticus) fishbone. Kappa-Carrageenan is a colloid extracted from various red algae. It is frequently used in food and pharmaceutical industry. The collected tilapia fishbone was washed and boiled, dried and pulverized. These two materials were used to produce a composite membrane and used as a filter media.

\section{Materials and methods}

\subsection{Materials and reagents}

The main raw materials were kappa-carrageenan and tilapia fishbone. The kappa-carrageenan was obtained from the left-over carrageenan used from a carrageenan manufacturer in Carmona, Cavite. The fishbone used from tilapia obtained from Talisay, Batangas.

The reagents used were distilled water from the chemistry laboratory of Adamson University; Glutaraldehyde used as the cross linker for the carrageenan; Isopropanol used for pretreatment of carrageenan; Sodium hydroxide used alkalize the carrageenan solution; Monochloroacetic acid used for the carrageenan solution and ethanol used to terminate the oxygenation of the carrageenan solution.

\subsection{Experimental set-up}

The collected kappa-carrageenan was screened using vibratory screener using mesh 60 to get a particle size of $0.250 \mathrm{~mm}$. For 200 grams of kappa-carrageenan powder

*Corresponding author: jerry.olay@ adamson.edu.ph 
and $50 \mathrm{ml}$ isopropanol were mixed and continuously stirred for 30 minutes. During the continuous stirring, 50 $\mathrm{ml}$ of sodium hydroxide and water were added and left for 1 hour to alkalize. $2 \mathrm{ml}$ of monochloroacetic acid was added in the mixture in gradual drop and left for 5 hours in the oven with an operating temperature at $50^{\circ} \mathrm{C} .50 \mathrm{ml}$ of ethanol was added after 5 hours. The mixture was filtered and washed up to 3 times to be able to get the solid particles that were formed. These solid particles were dried in desiccators for 1 day. The collected tilapia fishbone was washed and boiled carefully for 5-7 minutes and dried at $100{ }^{\circ} \mathrm{C}$ in an oven dryer to remove the moisture. After the removal of water content in a fishbone, it was further dried at $500-700{ }^{\circ} \mathrm{C}$ to prepare for the fishbone for the pulverization process. Using mortar and pestle, the samples were crush and screen using vibratory screener to be able to get a mesh 60 particle size. The k-carrageenan and tilapia fishbone were weighed according to the various blend-weight ratios of 4 composites solutions. Each solution were prepared based on $3 \mathrm{~g}$ total polymer weight.

Table 1. K-Carrageenan-Fishbone Composite Blend Weigh Ratio

\begin{tabular}{ccc}
\hline \multirow{2}{*}{$\begin{array}{c}\text { Composite } \\
\text { Number }\end{array}$} & \multicolumn{2}{c}{ Blend Weight Ratio (wt. \%) } \\
Kappa Carrageenan & Fishbone \\
\hline 1 & & $0 \%$ \\
2 & $100 \%$ & \\
3 & $90 \%$ & $10 \%$ \\
4 & $80 \%$ & $20 \%$ \\
5 & $70 \%$ & $30 \%$ \\
& $60 \%$ & $40 \%$ \\
\hline
\end{tabular}

Table 1 showed the different composite with different kappa-carrageenan-tilapia fishbone weight ratio. Each composite with a various blend-weight ratio was dissolved in a $100 \mathrm{ml}$ distilled water $\left(70^{\circ} \mathrm{C}\right)$ using a magnetic stirrer for one hour to prepare film-forming solutions. Diluted glutaraldehyde (50\% gram GA/gram GA solution) was added in the composite solutions during the film forming process. Each solutions were cast into the petri dishes. After casting, the composite solutions were dried for 3 consecutive days in an oven dryer at $50-65{ }^{\circ} \mathrm{C}$ for a total of 6 hours each day. The dried membranes on the petri dishes were submerged for one hour in the distilled water. The expanded or swollen membrane was then been spontaneously peeled-off from the petri dishes. Each composite membrane produced undergone a series of test to determine the following; calcium content (using ICP MS-Inductively Coupled Plasma Mass Spectrometry trace metal analysis), mechanical strength and elastic resistance (using Force Gauge Chatillon DFE-50 Tensile Testing Machine), water permeability (filtration by gravity at 1 atm pressure), and mean pore diameter size (by SEMScanning Electron Microscopy). With the exception of the water permeability, all of the physical properties were evaluated in a packaging corporation in Silang, Cavite.

\section{Results and discussion}

\subsection{Calcium Concentration of the composite membrane}

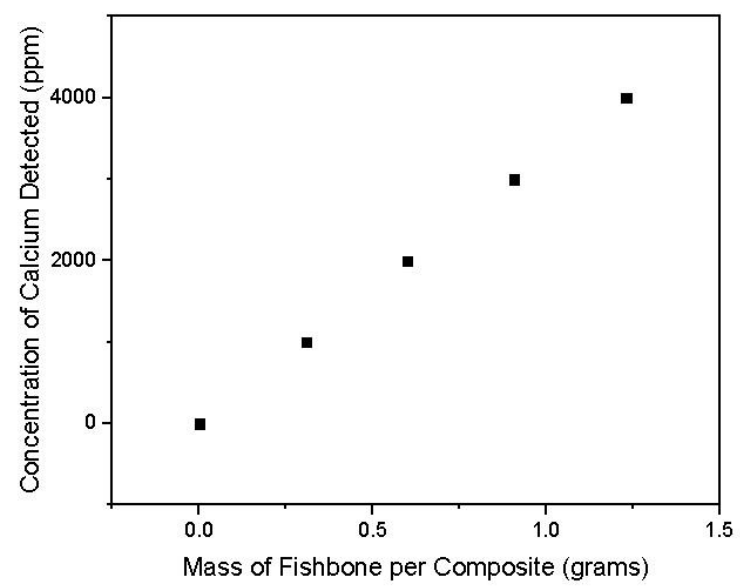

Figure 1. Calcium Concentration and Fishbone Weights per Composite

Figure 1 showed the variation of calcium concentration content in each composite made with varying fishbone weights. Composite no.1 served as the control sample (100\% k-carrageenan). Using Inductively Coupled Plasma Mass Spectrometry (ICP-MS) trace metal analysis, calcium content in each composite was determined. For composites 2, 3, 4 and 5, fishbone weights were $0.3,0.6,0.9$ and $1.2 \mathrm{~g}$ respectively. The test showed that for each composite, concentrations of calcium were detected as follows; 1000, 2000, 3000 and $4000 \mathrm{ppm}$. Based from the result, the higher the amount of calcium concentration in the composite, the more hydroxyl apatite content, which is a natural ceramic material formed from the bonding of calcium apatite and hydroxyl group [5].

\subsection{Mechanical and physical characteristics of composite membrane}

\subsubsection{Mechanical strength}

Mechanical Strength was one of the major characteristics of a membrane that should was evaluated since it affects the performance. Each membrane was subjected to mechanical strength test for evaluation. The test specimen prepared had a specific surface area ranging from 23.0 to $27.7 \mathrm{~m}^{2} / \mathrm{g}$ with thickness ranging from 15.8 to 26.5 microns.

Figure 2 shows the average tensile strength measured using Force Gauge (Chatillon DFE-50) for each membrane composite. For membrane composite 1, 2, 3, 4 and 5 it was shown that the measured tensile strength was $0.907,0.918$, and $1.434,1.677$ and $1.700 \mathrm{kgf} / 10 \mathrm{~mm}$ respectively. 


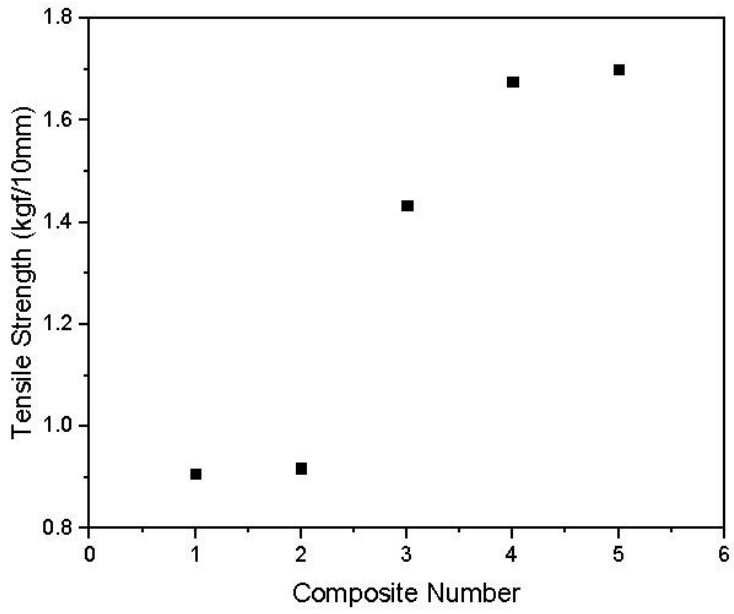

Figure 2. Tensile Strength of Composite Membrane

Composite no. 5 has the highest measured tensile strength and composite no. 1 have the lowest value (100\% carrageenan and control specimen). Based from the result, the amount of glutaraldehyde used as a crosslinking agent between the carrageenan and fishbone composite influenced the tensile strength because it facilitated the configuration or formation of linkage from its hydroxyl group [6].

\subsubsection{Elastic resistance}

Elastic Resistance or the elasticity of the composite membrane was also an important factor as tensile strength in its performance as a filtering media. In this research, each membrane composite was subjected to elastic resistance test for evaluation. The elastic resistance of the test specimen were taken simultaneously with the tensile strength.

Figure 3 shows the average elastic resistance measured using Force Gauge (Chatillon DFE-50) for each composite produced. For composite 1, 2, 3, 4 and 5 it was measured that it has an elastic resistance of $2.72,2.53$, $2.44,2.26$, and $2.17 \mathrm{kgf} / 10 \mathrm{~mm}$ respectively. Based from the result, the composite no. 1 which was the control, yielded the highest value. As observed, as the amount of carrageenan decreases in the composite, the elastic resistance decreased. The polysaccharide backbone chain possessed by the kappa- carrageenan that marked as an elastic and gelling component for in the composite membrane was affected by the particles of the fishbone added. In this case, the highest average elastic resistance was favored for filtration [6].

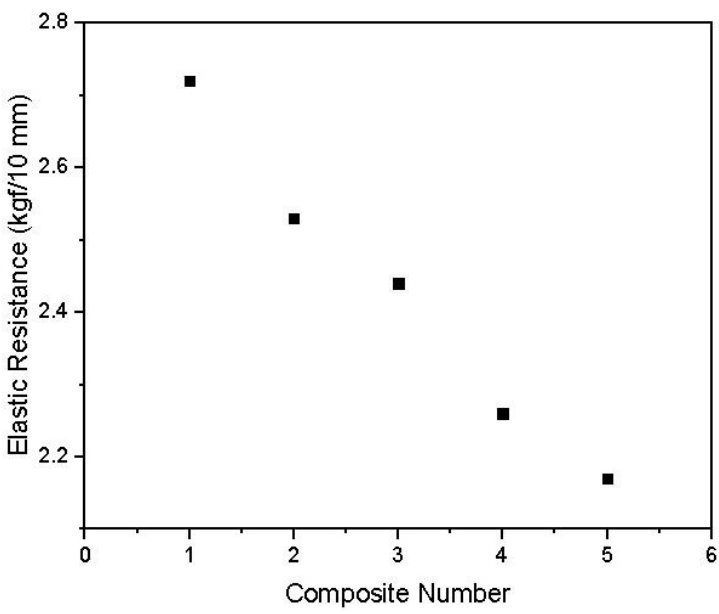

Figure 3. Elastic Resistance of Composite Membrane

\subsubsection{Water permeability}

Water permeability of the membrane was an important factor to determine the effectiveness and performance of a membrane as a filtering media, since it tells on how much water can it filter in each time. Table 2 shows the different water permeability factors of each composite. The water flux was computed using the equation (1).

$$
\text { Iv }=\frac{\text { Volume of water filtered }(\mathrm{m} 3)}{\text { Time of filtration }(\mathrm{s}) \times \text { Area of Filtration }(\mathrm{m} 2)}
$$

Table 2. Water Permeability Factors

\begin{tabular}{ccccc}
\hline $\begin{array}{c}\text { Compo- } \\
\text { sites }\end{array}$ & $\begin{array}{c}\text { Area } \\
\text { subjected } \\
\text { to } \\
\text { filtration } \\
\left(\mathrm{cm}^{2}\right)\end{array}$ & $\begin{array}{c}\text { Average } \\
\text { Volume of } \\
\text { Water } \\
\text { filtered }(\mathrm{mL})\end{array}$ & $\begin{array}{c}\text { Time } \\
\text { of } \\
\text { Filtra- } \\
\text { tion }(\mathrm{s})\end{array}$ & $\begin{array}{c}\text { Water } \\
\text { Flux } \\
(\mathrm{m} / \mathrm{s})\end{array}$ \\
\hline 1 & 19.63 & 192.37 & 1200 & 0.0000817 \\
2 & 19.63 & 164.93 & 1200 & 0.00007 \\
3 & 19.63 & 155.67 & 1200 & 0.0000663 \\
4 & 19.63 & 203.13 & 1200 & 0.0000862 \\
5 & 19.63 & 274.75 & 1200 & 0.000117 \\
\hline
\end{tabular}

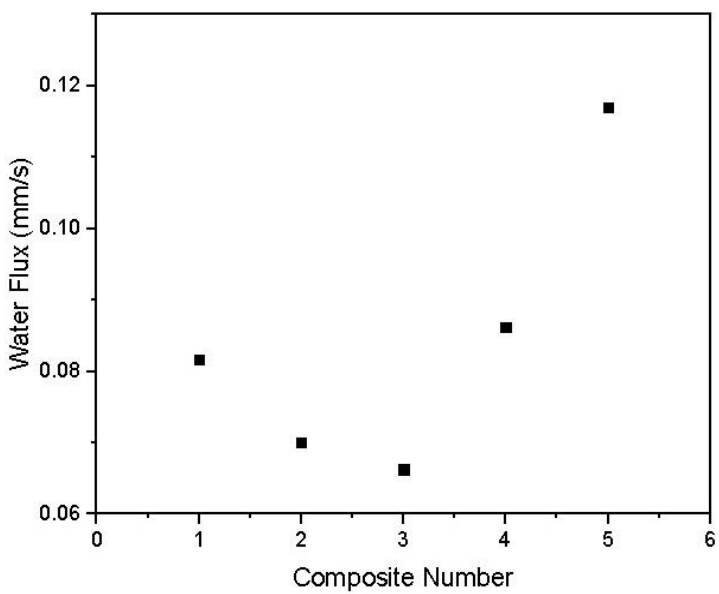

Figure 4. Water Permeability of Composite 
Figure 4 showed the average water flux computed for each composite produced. For composites 1, 2, 3, 4 and 5 , it was measured that it has the following water flux of $0.0817, \quad 0.0700,0.0663,0.0862$ and $0.117 \mathrm{~mm} / \mathrm{s}$ respectively. Composite no. 5 had the highest water flux while composite no 3 had the lowest water flux. The result was due to the pore size of membrane that affected how water passed through the filter. The composite with the highest water flux is favored which was a good characteristic for a filter medium [6].

\subsubsection{Pore size}

In membrane technology, pore size was the one of the important factor that influenced the performance of the membrane as a filter. Each membrane was subjected to Scanning Electron Microscopy (SEM) for evaluation. Most of the morphology of the test specimen were round as per SEM result.

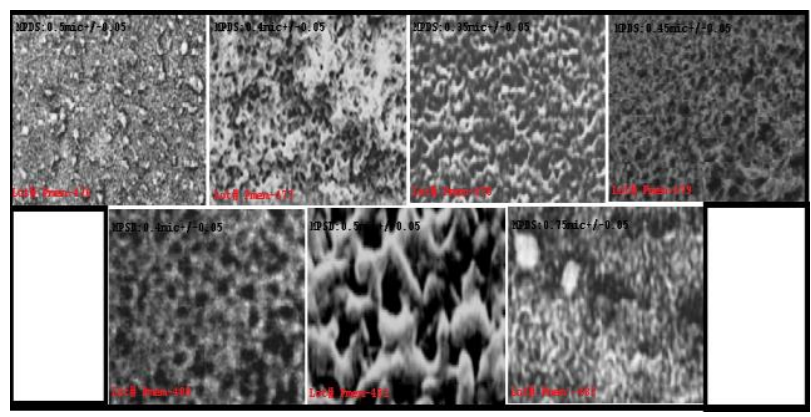

Figure 5. SEM images of Polymer Membrane Composites

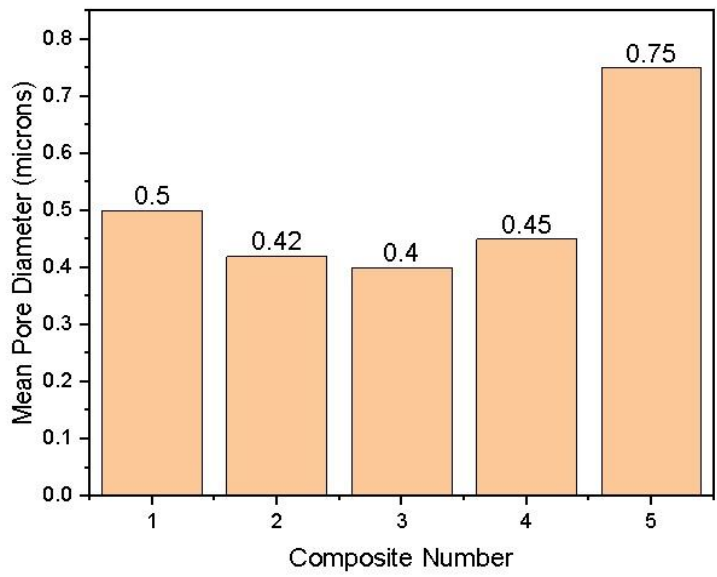

Figure 6. Mean Pore Diameter of Composite Membrane

Figure 6 showed the average mean pore diameter measured for each composite produced. For composites $1,2,3,4$ and 5, it was measured that it had the following mean pore diameter of $0.5,0.42,0.4,0.45$ and 0.75 microns respectively. Pore size of a membrane varied with the dependency on the optimum mixture of green based polymer with the material derivative of the composite [4]. The composite with the lowest mean pore diameter was favored for filtration. Composite no. 3 had the lowest mean pore diameter which was a good characteristic of a membrane used as a filter medium.

\subsection{Effects of the variation of composition with respect to its physical and mechanical properties}

Table 3 showed the P-value of the factors and interaction in comparison with the alpha values. The P-value for the composition factor, as seen on the table above, was lower compared the alpha value. Thus, the hypothesis that the variation of compositions of composite membrane was just the same with the other composite was rejected. Also, the P-value for mechanical and physical properties of polymer membrane is lower than 0.05 , indicating that the mechanical and physical properties factor was significant as well, regardless of the composition factor. The P-value for the interaction of composition and the properties was lower than the alpha-value. Therefore, there was a significant difference between the composition of composite membrane and its mechanical and physical properties.

Table 3. Summarized P-values of Variation

\begin{tabular}{|c|c|c|}
\hline Source of Variation & $\boldsymbol{\alpha}$-value & P-value \\
\hline Composition & 0.05 & $1.71 \mathrm{E}-20$ \\
\hline $\begin{array}{c}\text { Mechanical and Physical } \\
\text { Properties }\end{array}$ & 0.05 & $2.36 \mathrm{E}-65$ \\
\hline Interaction & 0.05 & $1.74 \mathrm{E}-35$ \\
\hline
\end{tabular}

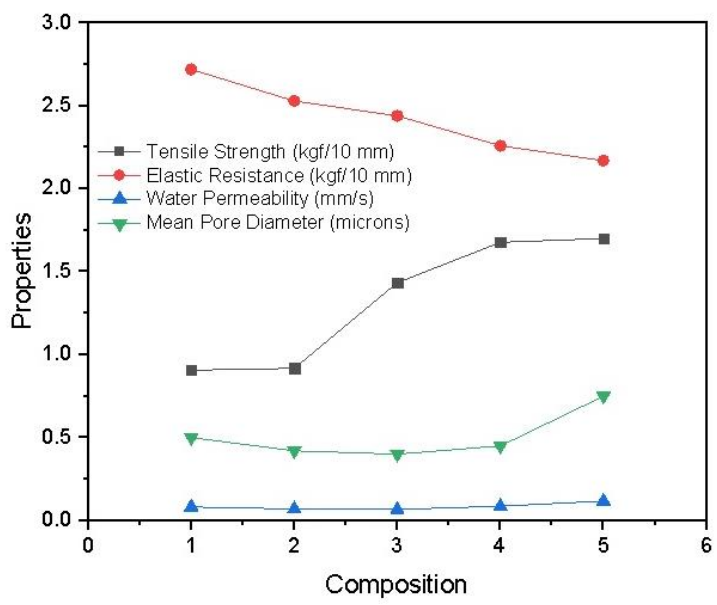

Figure 7. Variation of composition versus its properties

The significance of the factors composition and properties was shown in the Figure 7 . Based from the statistical analysis, as the percentage of the fishbone increased, the mechanical strength also increased, this was due to the metal (calcium) brought about by the fishbone as hydroxyl apatite [7]. On the other hand, the elastic resistance decreased as the mechanical strength increased. This was due to the polysaccharide backbone chain possessed by the kappa-carrageenan that marked it as an elastic component in the composite combination. In terms of pore size, there was a small difference between composite no.1 (control) with no. 4. Composite no. 5 has the highest pore size where this attributed with the greater percentage of fishbone in its composition. In terms with the water permeability, there was a gradual decrease form composite 1 up to 3 but achieved a higher amount of water flux with composite 5 . 


\section{Conclusion}

The overall aim of this research was to produce a composite membrane from waste kappa-carrageenan and tilapia fishbone. This study was able to produce a kappacarrageenan-fishbone composite membrane at different compositions based on weight percentages of $100 \%$ kappa-carrageenan, 90\% kappa -carrageenan and 10\% fishbone, $80 \%$ kappa-carrageenan and 20\% fishbone, $70 \%$ kappa carrageenan and 30\% fishbone, and $60 \%$ kappa carrageenan and $40 \%$ fishbone.

The mechanical and physical properties of the composite membrane were evaluated by the following parameters: mechanical strength, elastic resistance, pore size, and water permeability. The composite no. 5 with a ratio of $60: 40$ by weight of kappa-carrageenan and fishbone polymer membrane obtained the best results for mechanical strength and water permeability, while composite no 3 obtained the best results for pore size and elastic resistance respectively.

This research likes to acknowledge the support of the Chemical Engineering Laboratory of Adamson University.

\section{References}

[1] Available online at www.lenntech.com/membranetechnology.htm

[2] Marcano, J.G. S., Tsotsis, T. T., Catalytic Membranes and Membrane Reactors Wiley-VCH Verlag GmbH, Germany, 2002

[3] Lau, W. J., Ismail, A.F., Missdan, M.,Kassim, M.A. , A Recent Progress in thin film composite membrane: A Review, Desalination, vol. 289, pp. 190-199, 2012.

[4] Jumaah, Fatihah N., Mobaraka, Nadhratun N., Ahmad, Azizan, and Ramli, Nazaruddin, Characterization of 1-Carrageenan and its Derivative Based Green Polymer Electrolytes, AIP Conference Proceedings, 1572, Melville, NY, 2013, pp. 768-774

[5] Karunakaran, Ashvin Praveen, Filtration of Water through Nanostructures present in Carbonated Calcium Phosphate Bio Ceramic Powder from Fishbone, APEC Youth Scientist Journal, Vol.7 No.2, 2015, pp.315-324.

[6] Wu, Peng and Imai, Masanao, Food Polymer Pullulan-Carrageenan Composite Membrane performed Smart Function both on Mass Transfer and Molecular Size Recognition, Desalination and Water Treatment, Vol. 34, October 2011, p. 239-245

[7] Huang, Yi-Cheng and Chu, Hao-Wen, Using Hydroxyapatite from Fish Scales to prepare Chitosan/Gelatin/hydroxyapatite Membrane: Exploring Potential for Bone Tissue Engineering, Journal of Marine Science and Technology, Vol.217 (6), December 2013, pp. 716-722. 